\title{
Interaction kinetics of peptide lipids-mediated gene delivery
}

Yinan Zhao ${ }^{1}$, Tianyi Zhao ${ }^{2}$, Yanyan Du ${ }^{1}$, Yingnan Cao ${ }^{1}$, Yang Xuan ${ }^{1}$, Huiying Chen ${ }^{1}$, Defu Zhi ${ }^{1}$, Shutao Guo ${ }^{3 *}$, Fangli Zhong ${ }^{4^{*}}$ and Shubiao Zhang ${ }^{1^{*}}$

\begin{abstract}
Background: During the course of gene transfection, the interaction kinetics between liposomes and DNA is speculated to play very important role for blood stability, cellular uptake, DNA release and finally transfection efficiency.

Results: As cationic peptide liposomes exhibited great gene transfer activities both in vitro and in vivo, two peptide lipids, containing a tri-ornithine head (LOrn3) and a mono-ornithine head (LOrn1), were chosen to further clarify the process of liposome-mediated gene delivery in this study. The results show that the electrostatically-driven binding between DNA and liposomes reached nearly 100\% at equilibrium, and high affinity of LOrn3 to DNA led to fast binding rate between them. The binding process between LOrn3 and DNA conformed to the kinetics equation: $y=1.663631 \times \exp (-0.003427 x)+6.278163$. Compared to liposome LOrn1, the liposome LOrn3/DNA lipoplex exhibited a faster and more uniform uptake in HeLa cells, as LOrn3 with a tri-ornithine peptide headgroup had a stronger interaction with the negatively charged cell membrane than LOrn1. The efficient endosomal escape of DNA from LOrn3 lipoplex was facilitated by the acidity in late endosomes, resulting in broken carbamate bonds, as well as the "proton sponge effect" of the lipid.
\end{abstract}

Conclusions: The interaction kinetics is a key factor for DNA transfection efficiency. This work provided insights into peptide lipid-mediated DNA delivery that could guide the development of the next generation of delivery systems for gene therapeutics.

Keywords: Peptide lipids, Interaction kinetics, Uptake, Gene release, Gene delivery

\section{Introduction}

Gene therapy is the technology to transfer exogenous normal gene into the target cells to correct or compensate disease caused by genetic defects and anomalies, so

\footnotetext{
*Correspondence: stguo@nankai.edu.cn; fanglizhong@sina.co; zsb@dlnu. edu.cn

${ }^{1}$ Key Laboratory of Biotechnology and Bioresources Utilization of Ministry of Education, College of Life Sciences, Dalian Minzu University, Dalian 116600, China

${ }^{3}$ Key Laboratory of Functional Polymer Materials of Ministry of Education, State Key Laboratory of Medicinal Chemical Biology and Institute of Polymer Chemistry, College of Chemistry, Nankai University, Tianjin 300071, China

${ }^{4}$ School of Chemistry and Pharmaceutical Engineering, Jilin Institute of Chemical Technology, Jilin 132022, China

Full list of author information is available at the end of the article
}

as to achieve the aim of treating disease [1, 2]. Peptidebased cationic lipids are promising nonviral tools for nucleic acid delivery into cells, e.g., plasmids DNA for transfection and siRNA for gene silencing [3-6]. They have shown to possess properties that are superior to those of other cationic lipids, such as good biodegradability, excellent biocompatibility, and the ability to target to cells [7-10]. The primary requirements for a successful peptide lipid vector are the ability to compact nucleic acid, to protect it against degradation, and to deliver it into the cell with efficiency and specificity. In addition, liposome/DNA lipoplex must bind to the cell surface, enter cells by adsorption-mediated endocytosis, and accumulate in endosomes [11, 12]. Finally, lipoplexes 
escape from the endosomes and release the nucleic acid for successful gene expression [13-15].

In the process of delivering nucleic acid by peptide liposome vectors, the interaction between them has a very important effect on cellular uptake, nucleic acid release, and DNA or siRNA escape from endosomes, which are necessary for efficient gene transfer. Therefore, a perfect balance in the interaction between the liposomes and nucleic acid is required for the lipoplexes to behave as successful gene delivery vectors. Without knowledge of the interaction of liposomes with gene and the limiting barriers involved, it will be difficult to take a rational approach to develop improved methods of gene transfer and to test specific hypotheses related to cellular and molecular mechanisms.

Researchers have used the model of quaternary ammonium lipid to corroborate the interaction between lipid and DNA. They found that the binding between lipids and DNA is accomplished by electrostatic attraction [16]. When DNA is mixed with liposomes composed of cationic lipids and helper-lipids, the resulting lipids/DNA lipoplexes may consist of a multilamellar strucutre $\left(\mathrm{L}_{\alpha}^{\mathrm{C}}\right)$ comprising DNA monolayers sandwiched between lipid bilayers. $\mathrm{L}_{\alpha}^{\mathrm{C}}$ structure could be converted to an inverted hexagonal $\left(\mathrm{H}_{\|}^{\mathrm{C}}\right)$ structure by adjusting the helper-lipid, then the $\mathrm{H}_{\|}^{\mathrm{C}}$ phase of lipids/DNA lipoplexes increase fusion of the lipoplex with the endosomal membranes in cells, and promote the DNA release into the cytoplasm, therefore increasing the transfection efficiency $[17,18]$. However, systemic and thorough interaction research between peptide-based lipids and DNA has hardly been performed by far. To better understand the gene transport process and develop additional applications of peptide lipids, we here report on the use of 1,2-bismyristyloxyamidopropyl tri-ornithine lipid (Orn3) for performing the research, and we used 1,2-bis-myristyloxyamidopropyl ornithine lipid (Orn1) as a control (their structures were shown in Fig. 1). Both were developed in our laboratory, and these lipids could efficiently transfer DNA and siRNA into tumor cells and tumors of mice, with little in vitro and in vivo toxicity [19].

We examined the kinetics of association and dissociation between lipids and DNA. The cellular uptake of the lipoplexes at the microscopic level was further analyzed. Furthermore, endosomal escape of DNA as mediated by lipoplexes was detected by live cell imaging of HeLa cells to further clarify the mechanism of lipid-mediated delivery of DNA. As shown in Fig. 2, the peptide lipids self-assembled into liposomes, which could bind DNA by charge interactions (Fig. 2a). The lipid in liposome was composed of acid-responsive carbamate bond, lipoplexes could disassemble under disassembly $\mathrm{pH}\left(\mathrm{pH}_{\text {dis }}\right)$ (Fig. $2 \mathrm{~b}$ ). After uptake by the cells, the lipoplexes could stimulate endosome escape due to proton buffering capacity. DNA was then released from lipoplexes under the late endosomes environment $(\mathrm{pH}=5.0 \sim 5.5)$, and efficient gene expression was obtained (Fig. 2c). In this process, we have found that the liposome LOrn3 with tri-peptide headgroup showed a significantly faster binding rate and higher affinity to DNA, and also showed higher efficiency for gene delivery. Hence, the revealed interaction kinetics of peptide lipids-mediated gene delivery in this study may serve guiding information in designing of gene carriers and studying mechanism of gene delivery.

\section{Materials and methods \\ Materials}

The peptide lipids Orn1 and Orn3 were synthesized by our lab. The co-lipid 1,2-Dioleoyl-3-trimetry-lammonium-propane chloride salt (DOPE) was purchased from Sigma-Aldrich (USA). The green fluorescent protein reporter gene (GFP-N1) was purchased from Promega Biotech Co. Ltd. (Beijing, China), and extracted in our lab. The labeled DNA with 6-carboxy-fluorescein (FAM) was purchased from GenPharma company (China). The DNA intercalating dyes GelRed $(10,000 \times$ in water),

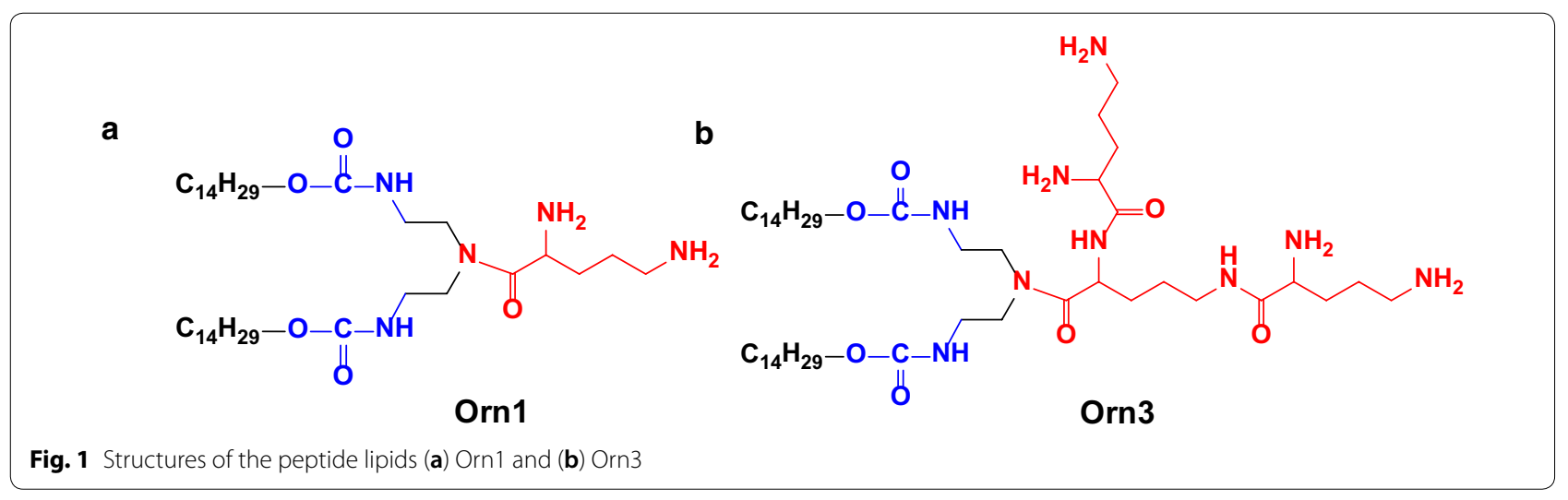




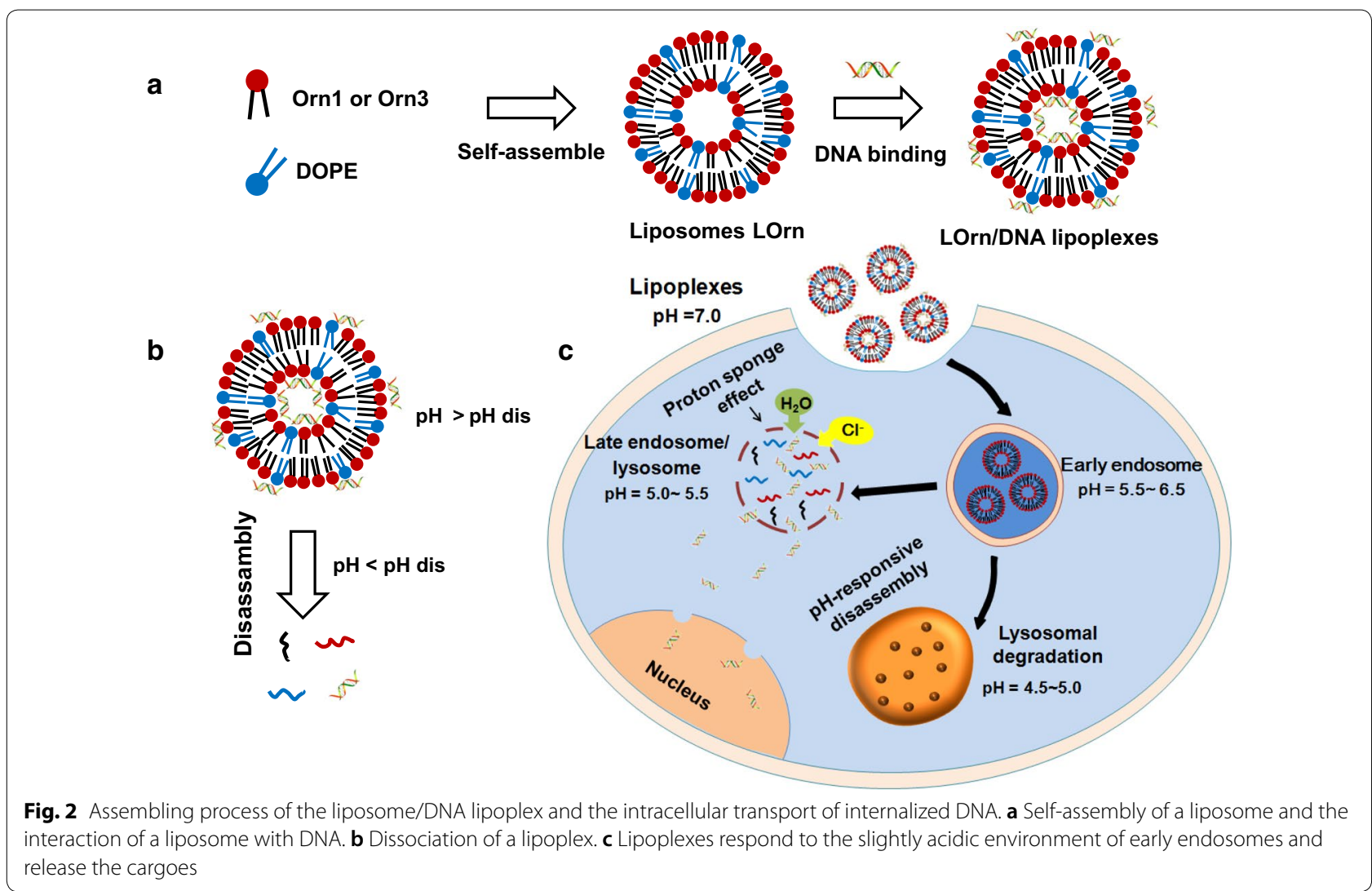

heparin, the liposome intercalating dye N-(7-Nitrobenz2-Oxa-1,3-Diazol-4-yl)-1,2-Dihexadecanoyl-sn-Glycero3-Phosphoethanolamine (NBD-PE), hoechst and Cy5 Nucleic Acid Labeling Kit were purchased from Biyotime biotechnology company (China), respectively. Trypsin was purchased from Gibco company (USA). All other chemicals were purchased from Alading (Shanghai, China). All water used was purified using a Milli-Q Plus 185 water purification system (Millipore, USA), giving a resistivity greater than $18 \mathrm{M} \Omega$.

\section{Liposomes preparation and characterization}

To prepare liposomes, peptide lipid and DOPE at the molar ratio of 1:1 were dissolved in $1 \mathrm{~mL}$ of chloroform in a glass vial; the solvent was removed under a stream of nitrogen gas, followed by high-vacuum desiccation. The dry lipid film was resuspended in $1 \mathrm{~mL}$ distilled water to give liposomes in a concentration of $1 \mathrm{mg} / \mathrm{mL}$. Liposome solutions were subjected to several cycles of sonication in a bath sonicator and vigorous vortex mixing to form small vesicles. The average particle sizes and Zeta potential of liposomes were measured by dynamic light scattering (DLS, HORIBASZ-100, Japan). Twenty microliter of liposomes were diluted to $1 \mathrm{~mL}$ with distilled water, all measurements were performed with freshly prepared liposomes at $25^{\circ} \mathrm{C}$ and in triplicate.

\section{Cell culture}

The HeLa cell line was purchased from Stem Cell Bank, Chinese Academy of Sciences (Shanghai, China) and grown in Dulbecco's modified Eagle's medium (DMEM, Gibco, Inc., USA) media supplemented with $10 \%$ fetal bovine serum (Gibco, Inc.) and $1 \%$ antibiotic mixture. The cells were grown at $37{ }^{\circ} \mathrm{C}$ with $65 \%$ humidity.

\section{DNA transfection}

To prepare lipoplexes, cationic liposomes were mixed with GFP-N1 in DMEM at liposome/DNA charge ratios ( \pm ) from 1:1 to $8: 1$ and incubated for $30 \mathrm{~min}$ at room temperature. In vitro DNA transfection of lipoplexes was measured against HeLa cell. The cells were seeded at $5 \times 10^{5}$ cells/well in 24 -well plates. After $24 \mathrm{~h}$, medium was replaced with serum-free medium, and lipoplexes in medium were added to each well and incubated for $4 \mathrm{~h}$ at $37{ }^{\circ} \mathrm{C}$. The medium was then replaced with medium containing $10 \% \mathrm{FBS}$ and $1 \%$ antibiotics, and the cultures were maintained at $37{ }^{\circ} \mathrm{C}$ under $5 \% \mathrm{CO}_{2}$. The expression of green fluorescent protein was measured by an 
inverted fluorescence microscope (Olympus IX71, Japan) and a flow cytometry (Becton-Dickinson, Heidelberg, Germany).

\section{Agarose gel electrophoresis}

The interaction of peptide liposomes with DNA at different charge ratios was confirmed by gel retardation assay. The final volume of lipoplexes was adjusted to $20 \mu \mathrm{L}$, and then mixed with $6 \times$ loading buffer $(2 \mu \mathrm{L})$. The mixture was loaded onto a $1.2 \%$ agarose gel containing $3 \mu \mathrm{L} \mathrm{Gel-}$ Red. Electrophoresis was carried out in $1 \times$ TAE running buffer at $90 \mathrm{~V}$ for $50 \mathrm{~min}$, and DNA bands were visualised at UV light wavelength of $300 \mathrm{~nm}$ by a gel documentation unit (LongGene LG3000, China). The binding amounts of DNA by peptide liposomes (or DNA release rate) were calculated using software of gel documentation unit.

\section{Cytotoxicity of lipoplexes}

Cytotoxicity of lipoplexes was analyzed by the MTT assay against HeLa tumor cells. Cells were seeded in 96-well plates and incubated at $37^{\circ} \mathrm{C}$ under $5 \% \mathrm{CO}_{2}$ for $18-24 \mathrm{~h}$ to achieve about $80-90 \%$ confluence. The LOrn1/DNA and LOrn3/DNA lipoplexes were added to the cells and incubated at $37^{\circ} \mathrm{C}$ under $5 \% \mathrm{CO}_{2}$ for $12,24,48$ and $72 \mathrm{~h}$, respectively. MTT ( $20 \mu \mathrm{L}, 5 \mathrm{mg} / \mathrm{mL}, \mathrm{pH} 7.4)$ was added, and cells were incubated with MTT for $4 \mathrm{~h}$, following which $150 \mu \mathrm{L}$ DMSO was added to each well to dissolve the substrate. The absorbance at $570 \mathrm{~nm}$ was monitored by Microplate Reader (Sunrise Tecan, Australia). Cells without lipoplexes served as controls. Cell viability was expressed as a percentage of the control. Cell viability was calculated as $\left([\mathrm{Abs}]_{\text {sample }} /[\mathrm{Abs}]_{\text {control }}\right) \times 100 \%$.

\section{Steady-state fluorescence spectroscopic of DNA binded with liposomes}

To make the GelRed labeled DNA, the DNA stock solutions and GelRed stock solutions were mixed at the DNA and GelRed weight ratio of 10:1 in the phosphate buffer and equilibrated for $15 \mathrm{~min}$. To make the liposomes/DNA lipoplexes, the peptide liposomes LOrn1 and DNA were mixed at 4:1 charge ratio $( \pm)$, the ratio was 3:1 for LOrn3 and DNA. To a GelRed-DNA mixture in a quartz cuvette were added the desired amounts of peptide liposomes stock solutions. The excitation wavelength of GelRed was $300 \mathrm{~nm}$, the emission was $600 \mathrm{~nm}$. The excitation slit and emission slit were fixed at 1 and $0.8 \mathrm{~nm}$, respectively. The temperature was set at $25{ }^{\circ} \mathrm{C}$. Recorded the fluorescence intensity of system for every $5-10 \mathrm{~min}$.

\section{Small-angle X-ray scattering (SAXS)}

Small-angle X-ray scattering (SAXS) measurement was determined by an Anton Paar Saxesess mc2 instrument
$(\mathrm{Cu}-\mathrm{K} \alpha)$ (Anton Paar, Austria). The X-ray generator was operated at $40 \mathrm{kV}$ and $50 \mathrm{~mA}$. A sample-detector distance was $260 \mathrm{~mm}$ and the X-ray wavelength $(\lambda)$ was $0.1542 \mathrm{~nm}$. The sample was measured at $25^{\circ} \mathrm{C}$.

\section{Stopped-flow fluorescence spectroscopic of DNA binded with liposomes}

The kinetic measurements were carried out using a stopped-flow fluorescence spectroscopic (SX-20MV, Applied Photophysics Ltd, UK). DNA $(4 \mu \mathrm{g} / \mathrm{mL})$ and GelRed $(10,000 \times)$ were mixed and kept for $40 \mathrm{~min}$ to get DNA/GelRed complex. Liposomes (1 mg/mL) and DNA/ GelRed complex at the charge ratios $( \pm)$ of $3: 1$ or $4: 1$ were used for stopped-flow fluorescence spectroscopy, and the excitation monochromator was set to $510 \mathrm{~nm}$. During the experiment, two separate syringes of the stopped-flow were filled up with DNA-GelRed complex and liposome solutions, and in each run, an equal volume of both solutions was injected at once into the sample chamber. The emission spectra were monitored continuously both before $(t=0 \mathrm{~s})$ and after the injection.

\section{The release of DNA from lipoplexes with agarose gel electrophoresis}

The lipoplexes were prepared with the method of DNAbinding assay above. The LOrn1/DNA and LOrn3/ DNA lipoplexes were added to a given concentrations $(0.05-2.0 \mu \mathrm{g} / \mu \mathrm{L})$ of heparin dissolved in PBS and incubated for $30 \mathrm{~min}$ at room temperature [20]. The samples $(20 \mu \mathrm{L})$ were electrophoresed on $1.2 \%$ agarose gel at different incubation time. Small amount of LOrn1/DNA and LOrn3/DNA lipoplexes were incubated with $\mathrm{pH}$ 5.5 phosphate buffer for $1,4,12,24,36,48,60$ and $72 \mathrm{~h}$, respectively, and the resulting solution was assessed by agarose gel electrophoresis.

\section{Cellular uptake using flow cytometry}

Cellular uptake experiments were performed on HeLa cell line. LOrn1 and LOrn3 liposomes with the concentration range of $1.8 \mu \mathrm{g} / \mathrm{mL}$ were prepared (diluted by DMEM without FBS, $\mathrm{pH}$ 7.4). The liposome/FAM-DNA charge ratios $( \pm)$ were 4:1 and 3:1 for LOrn1 and LOrn3, respectively. HeLa cells were seeded in 24-well plates at a density of $10^{5}$ cells/well and incubated in complete cell medium for $24 \mathrm{~h}$. Then, the medium was removed and replaced with $400 \mu \mathrm{L}$ DMEM per well without FBS. The lipoplexes $(100 \mu \mathrm{L})$ were added and incubated at $37{ }^{\circ} \mathrm{C}$ for $4 \mathrm{~h}$, the final concentration of LOrn1 and LOrn3 in lipoplexes were $8 \mu \mathrm{g} / \mathrm{mL}$ and $6 \mu \mathrm{g} / \mathrm{mL}$, respectively. Cells were washed with PBS, trypsinized, and resuspended in PBS at a concentration of $1 \times 10^{6}$ cells $/ \mathrm{mL}$. Then that used Trypan Blue to quench FAM-DNA fluorescence outside of cells, a stock solution of $0.4 \%(w / w)$. Trypan 
Blue in water was prepared and added at $10 \%$ of the volume of the cell suspension immediately before measurement. Cells were detected and quantified by flow cytometry (Becton-Dickinson, Heidelberg, Germany). Data were processed using the Cflow Plus software (Becton-Dickinson) [21, 22].

\section{Cellular uptake and DNA release using CLSM}

Confocal laser scanning microscopy (CLSM) and TEM were used to investigate the intracellular transport of lipoplexes [23, 24]. Hoechst 33,342, NBD-PE and Cy5DNA were used here in order to detect the intracellular distribution of liposome/DNA lipoplexes. The LOrn1 (or LOrn3) liposome was mixed with NBD-PE ethanol solution at liposome/NBD-PE volume ratio of 20:1, and stored overnight at $4{ }^{\circ} \mathrm{C}$. Cy5-labeled DNA was prepared by use of the Label IT Cy5 Nucleic Acid Labeling Kit, according to the manufacturer's instructions. HeLa cells were seeded into 6-well plates, cells were transfected with liposome/DNA lipoplexes after $24 \mathrm{~h}$. After $30 \mathrm{~min}$, $2 \mathrm{~h}$ and $4 \mathrm{~h}$ of transfection, the treated cells were washed three times with $1 \times \mathrm{PBS}$ to remove residual free lipoplexes. Then $400 \mu \mathrm{L}$ fresh DMEM containing Hoechst 33,342 was added into each well to stain cells. Hoechst 33,342 was used to stain cell nuclei. At last, the treated cells were imaged by CLSM (Olympus FV1000, Japan). For study of intracellular DNA release, LysoTracker Red was used to stain lysosomes [25]. Cells were exposed to LOrn3 liposome for $30 \mathrm{~min}, 2$, and $4 \mathrm{~h}$, cells were washed 3 times with PBS, fixed with $2.5 \%$ formaldehyde $/ 2.5 \%$ glutaraldehyde in $0.1 \mathrm{M}$ sodium cacodylate buffer at room temperature, and kept at $4{ }^{\circ} \mathrm{C}$ overnight. Then, the samples were washed several times in water, then dehydrated in increasing concentrations of ethanol, infiltrated, and embedded in Spurr's low-viscosity medium. The samples were polymerized in a $60{ }^{\circ} \mathrm{C}$ oven for 2 days. Ultrathin sections were cut in a Leica Ultracut microtome, stained with lead citrate in a Leica EM stainer (Leica, EM FC7 UC7, USA), and examined in a transmission electron microscope (JEM-2100, Carl Zeiss, Japan).

\section{Statistical analysis}

All data were expressed as mean \pm standard deviation (SD) for three independent tests. Differences between groups were analyzed using one-way analysis of variance (ANOVA) followed by LSD's multiple comparison post hoc test. The results were considered statistically significant when $p<0.05(" p<0.05 ; " p<0.01 ; " p<0.001)$.

\section{Results and discussion}

\section{Characterization of liposomes}

Our previous study showed that peptide liposomes with tri-ornithine as the headgroup (LOrn3) could transfer
DNA and DNA very efficiently into tumor cells, such as NCI-H460 and Hep-2 cells. Also, it was able to deliver combined DNAs against c-Myc and VEGF for silencing distinct oncogenic pathways in lung tumors of mice, with little in vitro and in vivo toxicity [19]. To develop further applications of peptide lipids for gene delivery, a comparison with the lipid LOrn1, with one ornithine as the headgroup, was used to obtain detailed information about the interaction between peptide lipids and genes and the intracellular transport process. Our previous study showed that liposomes had the highest transfection efficiency with equivalent molar ratio of DOPE, accordingly the liposomes LOrn1 and LOrn3 were prepared with DOPE as a co-lipid at a molar ratio of 1:1. Figure 2a shows that the sizes were around $80 \mathrm{~nm}$ for both liposomes LOrn1 and LOrn3 (Fig. 3a). And the Zeta potentials of LOrn1 and LOrn3 were $30 \mathrm{mV}$ and $45 \mathrm{mV}$ respectively (Fig. 3b), which ensured the stability of the liposomes. The morphological characteristics were visualized by transmission electron microscopy (TEM) (Fig. 3c). Images obtained from LOrn1 and LOrn3 liposomes revealed well-defined spheres structures, regularly shaped and uniformly distributed.

\section{DNA transfection in vitro}

In our previous work, we reported that peptide liposomes delivered DNA and siRNA into various tumor cells with Lipofectamine 2000 as control. We discovered that the transfection efficiency of liposomes had a similar performance in HeLa, Hep-2, A549, MCF-7, H460 cells, though HeLa and Hep- 2 cells showed higher transfection efficiency. Therefore, to evaluate further transfection efficiency of the two liposomes, we used them to deliver the GFP into HeLa and Hep-2 cells. The expression levels of GFP are shown in Additional file 1: Figure S1, Fig. 4a, b after the qualitative measurement of fluorescence and the quantitative determination of the fluorescent intensity. The charge ratios had a great impact on DNA transfection, LOrn1 and LOrn3 possessed the highest transfection efficiency at charge ratios of 4:1 and 3:1, respectively. And the expression of GFP of the LOrn3-treated cells was significantly stronger than that of the LOrn1-treated cells at the charge ratios from 1:1 to 8:1 in two cells. And the transfection efficiency against HeLa cell was higher than that against Hep- 2 cells. Therefore, we chose HeLa cells to do the subsequent experiments. And it's noted that LOrn1/DNA and LOrn3/DNA lipoplexes had much lower toxicity to HeLa cells at charge ratios of $4: 1$ and $3: 1$, respectively. The cell viability was also over $80 \%$ after treatment with lipoplexes for $72 \mathrm{~h}$ (Additional file 1: Fig. S2).

Then, we investigated the ability of liposomes to complex DNA at charge ratios from $0.5: 1$ to $8: 1$, findings 
in the gel retardation assay revealed that DNA binding amount increased with the charge ratios increase. LOrn1 and LOrn3 completely bound DNA at charge ratios more than 4:1 and 3:1, respectively (Fig. 4c, d). Meanwhile, the Zeta potentials of lipoplexes were detected, the results show that their Zeta potentials increased from negative to positive with the increase of charge ratios (Fig. 4e). For LOrn1 and LOrn3, the values became positive just at charge ratios of $4: 1$ and $3: 1$, respectively, corresponding with the charge ratios of complete DNA complex, these results accorded well with the above gel retardation assay results. This study indicates that LOrn3 was more effective than LOrn1 in terms of the interaction with DNA. In addition, synchrotron small-angle $x$-ray scattering (SAXS) was used to analyze the structures of the liposome/DNA

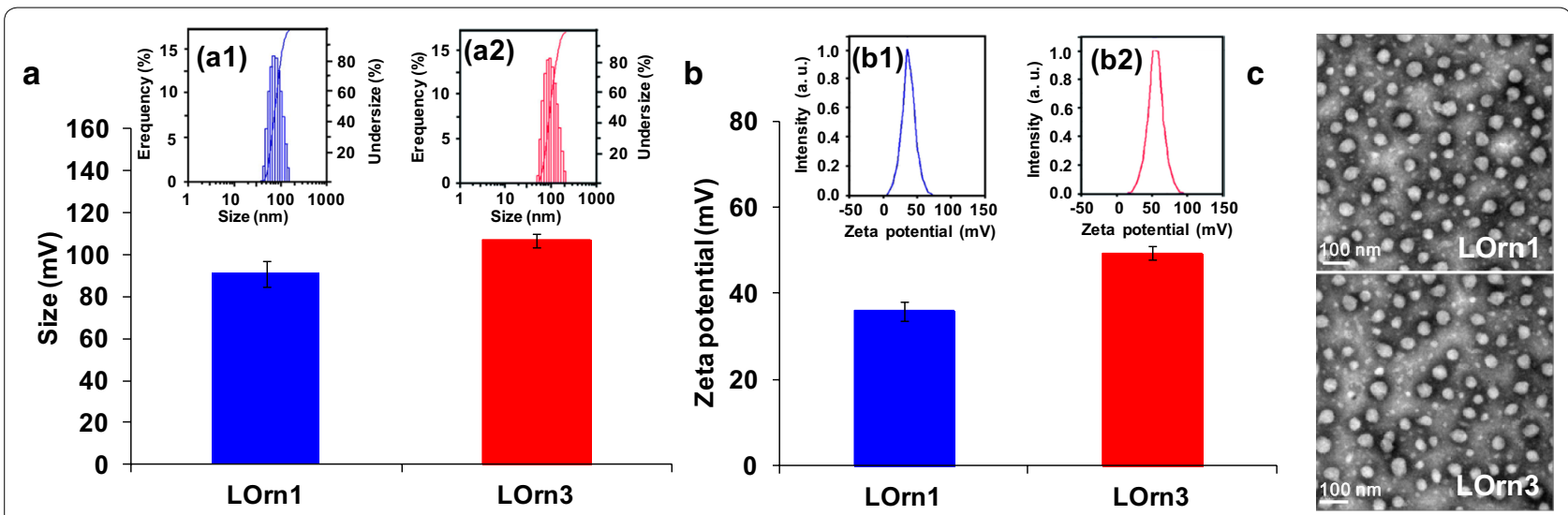

Fig. 3 Characterization of liposomes. a Particle size and (b) Zeta potential of liposomes measured by using a ZetaSizer. Particle size distribution of LOrn1 and LOrn3 is shown in (a1, a2), the PDI of LOrn1 and LOrn3 was $0.102 \pm 0.014$ and $0.097 \pm 0.006$, respectively. Zeta potential distribution of LOrn1 and LOrn3 is shown in (b1, b2). c Morphology of the particles measured by TEM, the diameters of Lorn1 and LOrn3 were $42.85 \pm 16.3 \mathrm{~nm}$ and $44.16 \pm 12.07 \mathrm{~nm}$, respectively (voltage: $100 \mathrm{kV}$ )

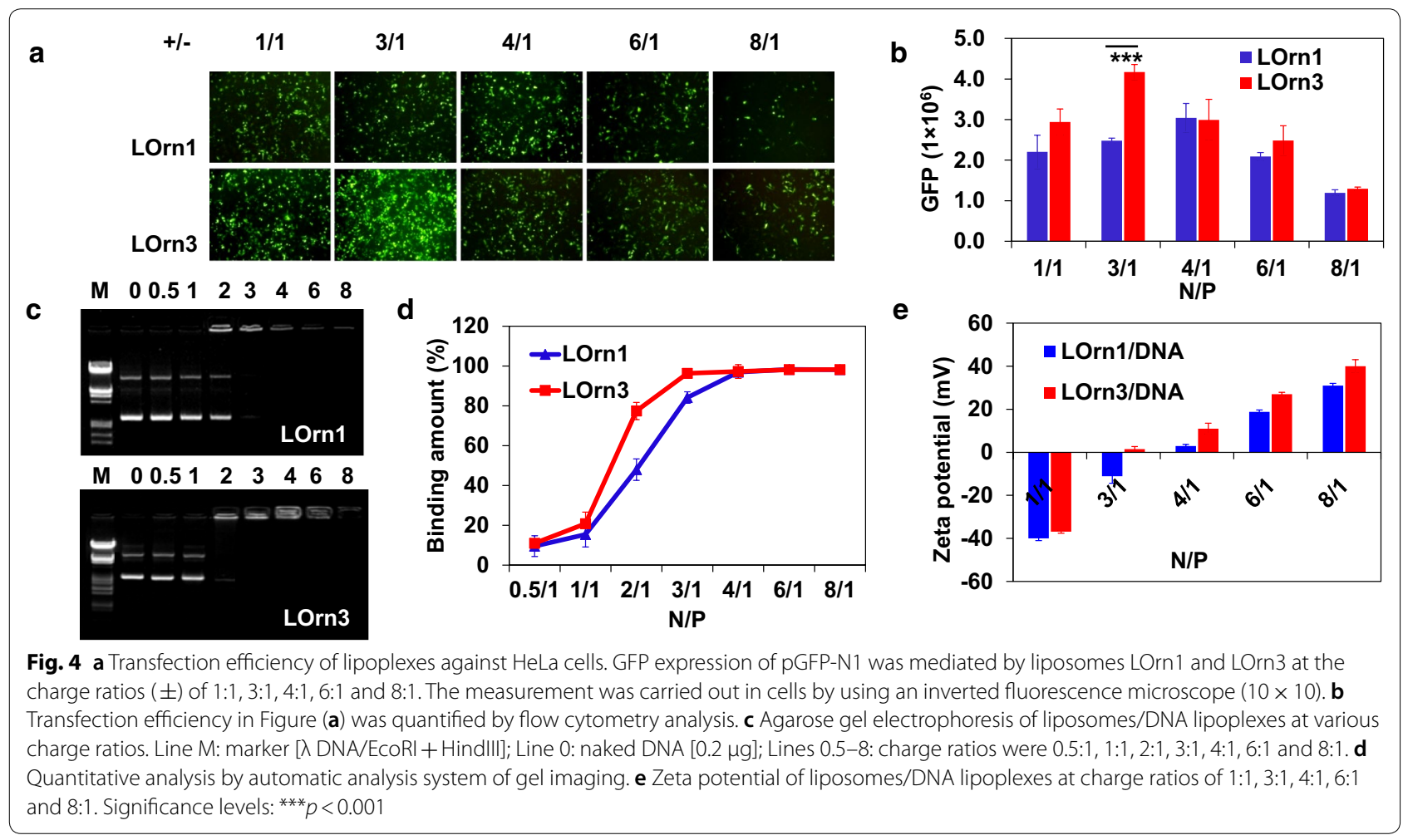


lipoplexes, and the liposome was composed of peptide lipid (LOrn1 or LOrn3) and DOPE at ratio of 1:1. For LOrn1/DNA and LOrn3/DNA lipoplexes, though the $\mathrm{L}^{\mathrm{C}}{ }_{\alpha}$ and $\mathrm{H}^{\mathrm{C}}$ structures coexisted (Additional file 1: Fig. S3), LOrn3/DNA lipoplex formed more $\mathrm{H}^{\mathrm{C}}{ }_{\|}$structures than LOrn1/DNA, which was considered as one reason for higher transfection efficiency of LOrn3. SAXS scans showed sharp peaks at $q_{100}=0.106 \AA^{-1}$ and $q_{200}=0.239 \AA^{-1}$ from the lamellar periodic structure $\left(d=2 \pi / q_{100}=59.27 \AA\right)$ and at $q_{10}=0.126 \AA^{-1}$ from hexagonal structure. The peptide lipid-DOPE bilayer thickness was $d \mathrm{~m}=40 \AA$ [17], the water gap between bilayer was $19.27 \AA(d \mathrm{w}=d-d \mathrm{~m})$. While the hexagonal lattice constant $a$ is $57.58 \AA\left(a=4 \pi /(3)^{0.5} q_{10}\right)$. The middle broad peak at $q_{11}=0.186 \AA^{-1}$ was due to the one-dimensional array of DNA chains, with the spacing between the DNA strands $\left(d_{\mathrm{DNA}}=2 \pi / q_{11}\right)$, which demonstrated that the DNA was able to be inserted between cationic lipid bilayers (thickness is $d \mathrm{w}$ ). During this process, DOPE is a fusogenic phospholipid that presents a super synergistic effect when used with cationic liposomes, decreasing the cytotoxicity imposed by the cationic lipids, destabilizing lipid bilayers by increasing the fusion with the cellular and endosomal membranes and allowing the release of DNA into the cytosol. Lipoplexes containing DOPE may facilitate the transition from lamellar to hexagonal structures promoting high gene transfection efficiency [17].

\section{Binding kinetics of liposome with DNA}

To obtain detailed information about the interaction between liposomes and DNA, we utilized agarose gel electrophoresis and fluorescent resonance spectroscopy to examine the time-dependent interaction of cationic peptide liposomes with DNA. As shown in Fig. 5a, $\mathrm{b}$, the binding amount of DNA significantly increased with time, and nearly 100\% DNA binding was reached after $30 \mathrm{~min}$ and $24 \mathrm{~min}$ for LOrn1 or LOrn3 liposomes, respectively. The data also demonstrated that the binding speed between liposome LOrn3 and DNA was faster than that between LOrn1 and DNA. The interaction between liposomes and DNA labeled with a nucleic dye (GelRed) was also monitored by fluorescence spectroscopy [26, 27]. GelRed fluoresces strongly when bound to DNA, and the displacement of GelRed from DNA by liposomes results in a decrease in fluorescence intensity that correlates with the amount of liposome binding DNA. The results show that the displacement of GelRed from DNA by liposome LOrn3 was much faster than that by LOrn1 (Fig. 5c). Moreover, the fluorescence intensity of GelRed/DNA was lower for LOrn3 when the displacement reached the equilibrium. The results substantiate the fact that the DNA binding of liposomes is faster at the initial stage, and LOrn3 has a stronger DNA binding ability than LOrn1. To further investigate the binding process of the two liposomes and DNA, a stopped-flow fluorimeter (SX-20MV, UK) setup was employed to monitor the formation of liposome/DNA lipoplexes using the fluorescent probe GelRed. The data indicate that GelRed fluorescence decreased much more rapidly for LOrn3 than LOrn1 (Fig. 5d), and the K values of LOrn1 and LOrn3 were 0.102795 and 0.003472 , respectively. By fitting the binding data, it was found that the binding process between liposomes and DNA conformed to the kinetics equations: $y=0.235141 \times \exp \quad(-0.102795 x)+3.461248$ and $y=1.663631 \times \exp \quad(-0.003427 x)+6.278163$ for LOrn1 and LOrn3, respectively. It means that the binding rate of LOrn3 and DNA was faster than that of LOrn1 and DNA. Then the binding affinity of liposomes to DNA was identified by using MicroScale Thermophoresis (MST) performed on the Monolith NT.115Pico system. In this method, the affinity of liposome to DNA is represented by $\mathrm{K}_{\mathrm{d}}$ value, which is the liposome concentration required to bind 50\% DNA [28, 29]. The dose response curves generated by titrating LOrn1 or LOrn3 liposomes to DNA solutions (Fig. 5e) show that the $\mathrm{K}_{\mathrm{d}}$ values of LOrn1 or LOrn3 were 0.296 and $0.136 \mu \mathrm{M}$, respectively. Therefore, we speculate that high affinity of LOrn3 to DNA led to fast binding rate between them, and then resulting in more efficient transfection. Therefore, we further evaluated the effect of interaction kinetics between the liposomes and DNA on the transfection efficiency. The expression of GFP gradually increased with binding time, and then reached to the maximum values at about $24 \mathrm{~min}$ and $32 \mathrm{~min}$ for LOrn 3 and LOrn 1 liposomes, respectively (Fig. 5f, g). At this time the interaction between liposomes and DNA also reached equilibrium (Fig. 5b, c). We confirmed that the interaction kinetics is a key factor for DNA transfection efficiency.

\section{Stability of lipoplexes in blood mimicked environment} Blood is rich in negatively charged macromolecules, such as serum protein and heparin, and these materials are known to dissociate DNA from lipoplexes by the interaction between liposomes and serum proteins [30-33]. Therefore, we first examined the effect of serum on the dissociation of lipoplexes, as shown in Fig. 6a, b, which depicts DNA release of lipoplexes in a medium with varying serum concentrations. A serum concentration of $10 \%$ had nearly no obvious effect on the stability of both lipoplexes, with a DNA release of about $2 \%$. With increasing serum concentrations, the stability of both lipoplexes decreased, and the LOrn1/DNA lipoplex showed much more DNA dissociation than the LOrn3/ DNA lipoplex. When the serum concentrations were greater than $25 \%$, the release of DNA from the LOrn1/ 


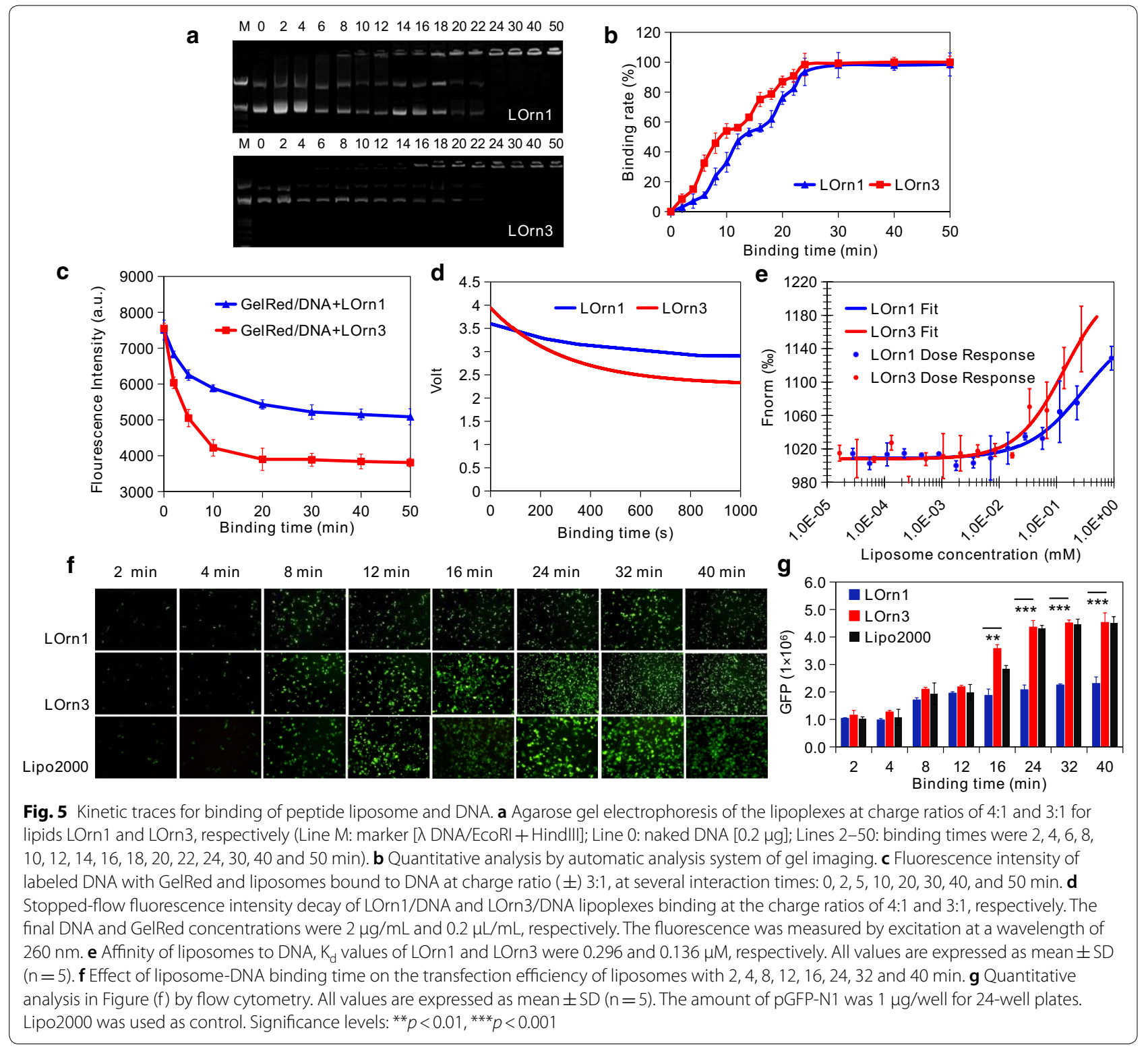

DNA lipoplexes increased to 20-35\%; in contrast, the percentage of DNA released from the LOrn3/DNA lipoplexes under the same conditions was much lower. Our previous study had already revealed that lower concentrations of serum $(5 \%$ or $10 \%)$ resulted in only a small reduction in transfection efficacy; with $20 \%$ serum, the transfection efficacy was slightly lowered [34]. Therefore, we hypothesize LOrn3 could well protect DNA in the lipoplexes from the interaction with serum when they are in the bloodstream.

Heparin sodium can competitively bind to liposomes from lipoplexes, which may lead to the dissociation of lipoplexes in blood. Therefore, the stability of lipoplexes was further examined by adding heparin sodium to simulate the blood environment. Figure $6 c, d$ showed that the brightness and the area of the bands corresponding to free plasmid DNA increased with the increase in heparin concentration. Heparin sodium affected the stability of the LOrn1/DNA lipoplex to a much greater extent than the LOrn3/DNA lipoplex in the concentration range of $0.1-2.0 \mu \mathrm{g} / \mu \mathrm{L}$. At a heparin concentration that is common in the blood $(0.1 \mu \mathrm{g} / \mu \mathrm{L})$, the release of DNA from the LOrn1/DNA lipoplex was $40 \%$, and it was $20 \%$ for the LOrn3/DNA lipoplex. Therefore, the LOrn3/DNA lipoplex could show great advantages over LOrn1/DNA when used for gene delivery. 
a

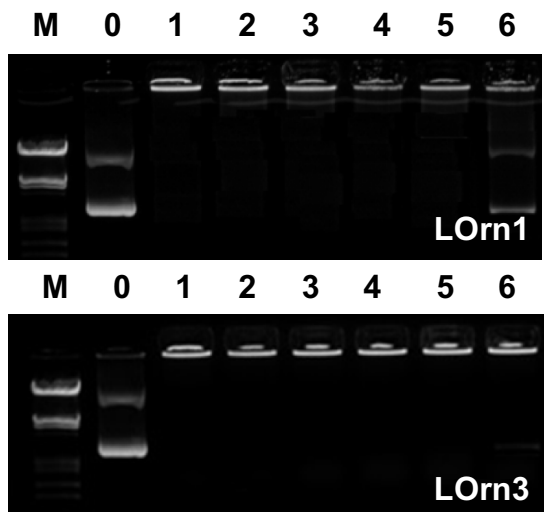

c

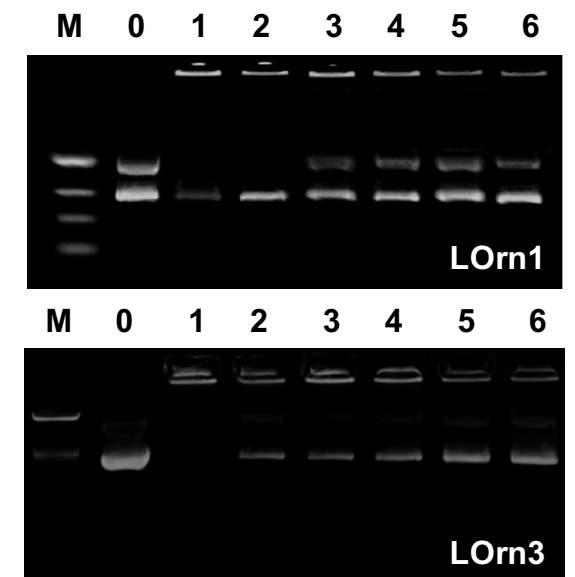

b

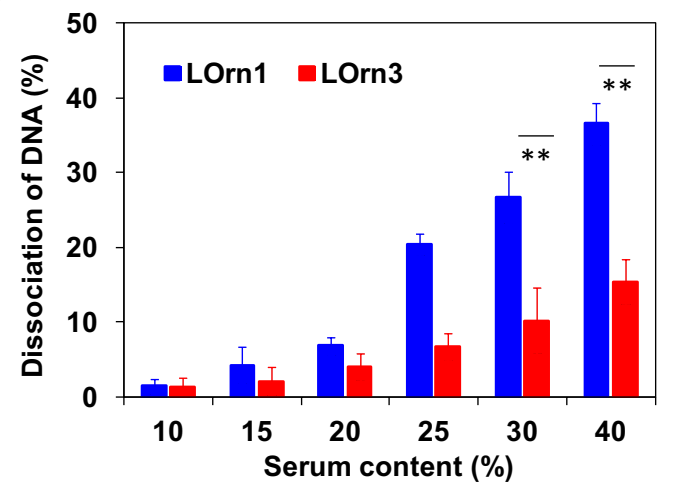

d

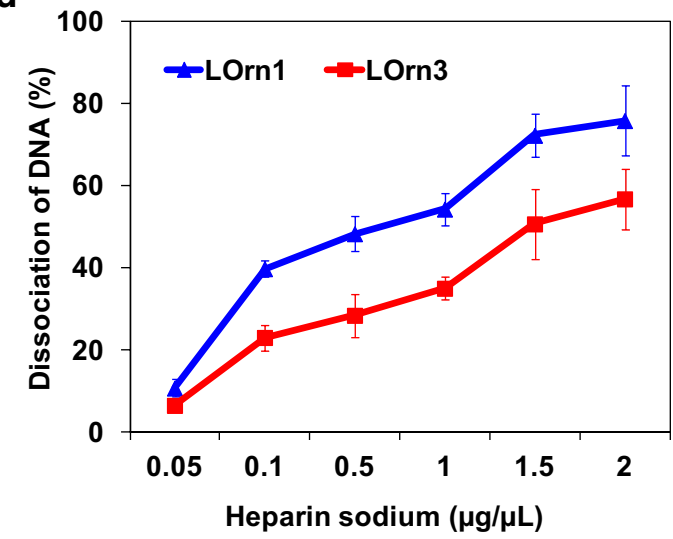

Fig. 6 Effect of serum and heparin sodium on the stability of LOrn1/DNA lipoplexes with a charge ratio of 4:1 and LOrn3/DNA lipoplexes with an charge ratio of 3:1. a Agarose gel electrophoresis of the lipoplexes at different serum contents (Lane M: marker; Lane DNA: free DNA; Lanes 1-6: serum contents of $10 \%, 15 \%, 20 \%, 25 \%, 30 \%$, and $40 \%$, respectively). $\mathbf{b}$ Quantitative analysis of the dissociation rate of lipoplexes in the present of serum. c Agarose gel electrophoresis of the lipoplexes at different concentrations of heparin sodium (Lane M: marker; Lane DNA: free DNA; Lanes 1-6: heparin sodium concentrations of $0.05,0.1,0.5,1.0,1.5$, and $2.0 \mu \mathrm{g} / \mu \mathrm{L}$, respectively). $\mathbf{d}$ Quantitative analysis of the dissociation rate of lipoplexes in the present of heparin sodium. Each bar represents the mean $\pm \operatorname{SD}(n=5)$. Significance levels: ${ }^{* *} p<0.01$

\section{Cellular uptake of lipoplexes}

Cellular uptake is required for successful transfection of genes. The cellular uptake of liposomes/FAM-DNA lipoplexes was first evaluated by FACS within $6 \mathrm{~h}$. As Trypan Blue could quench FAM-DNA fluorescence outside cells, it was used to differentiate internalized vs extracellular fluorescence markers. As displayed in Fig. 7a, the cellular uptake increased gradually with the transfection time; cellular uptake rates of LOrn1 and LOrn3 were about $73 \%$ and $94 \%$ after $4 \mathrm{~h}$, respectively. LOrn 3 showed a stronger interaction with the negatively charged cell membrane than LOrn1, so the LOrn3/DNA lipoplex could more easily cross the cell membrane, resulting in a higher cellular uptake rate compared with LOrn1/DNA. And we examined its morphological features at the ultrastructural level in HeLa cells incubated with LOrn3/DNA lipoplexes for different periods of time (Fig. 7b). The majority of LOrn3/DNA lipoplexes were found within early endocytic structures (endosomes) and multi-vesicular bodies in the early phase of uptake $(2 \mathrm{~h})$. They were detected within lysosomes $4 \mathrm{~h}$ after exposure of cells to LOrn3/DNA lipoplexes.

To further explore the kinetics of LOrn3/DNA lipoplex uptake, cellular uptake of the liposome/DNA lipoplexes was also investigated by laser scanning confocal microscope (LSCM) (Fig. 7c). As illustrated in the merged images, LOrn3/DNA lipoplexes displayed visible signals in the cytoplasm at $30 \mathrm{~min}$ post-transfection, but only a small amount of LOrn1/DNA lipoplexes were internalized into the cells. Though more LOrn1/DNA lipoplexes were localized in the cytoplasm at 2 and $4 \mathrm{~h}$ post-transfection, it was found that the fluorescent signals of LOrn3/DNA lipoplexes were distributed throughout the entire cytoplasm at $2 \mathrm{~h}$ posttransfection and mainly gathered around the nuclei at $4 \mathrm{~h}$ post-transfection. Compared to liposome LOrn1, 


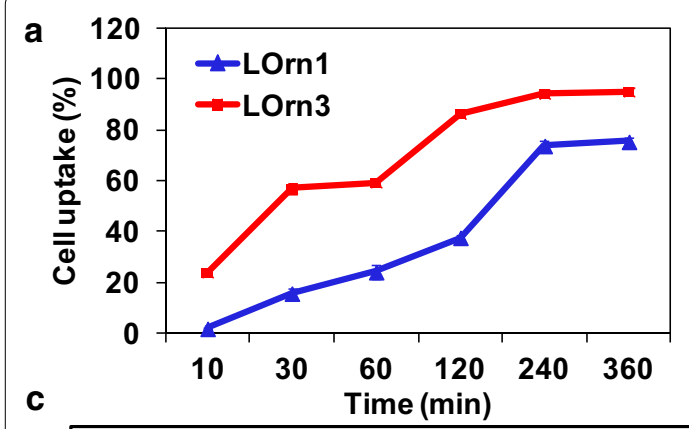

b

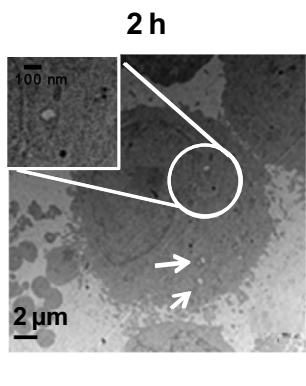

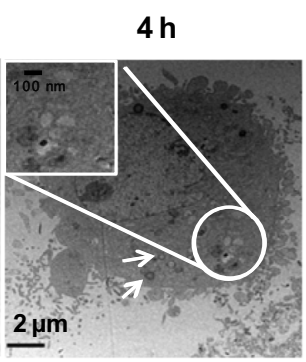

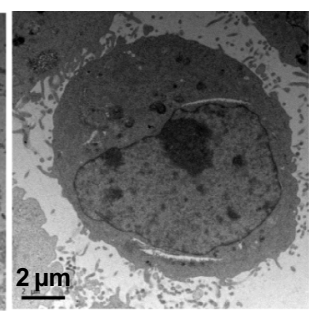

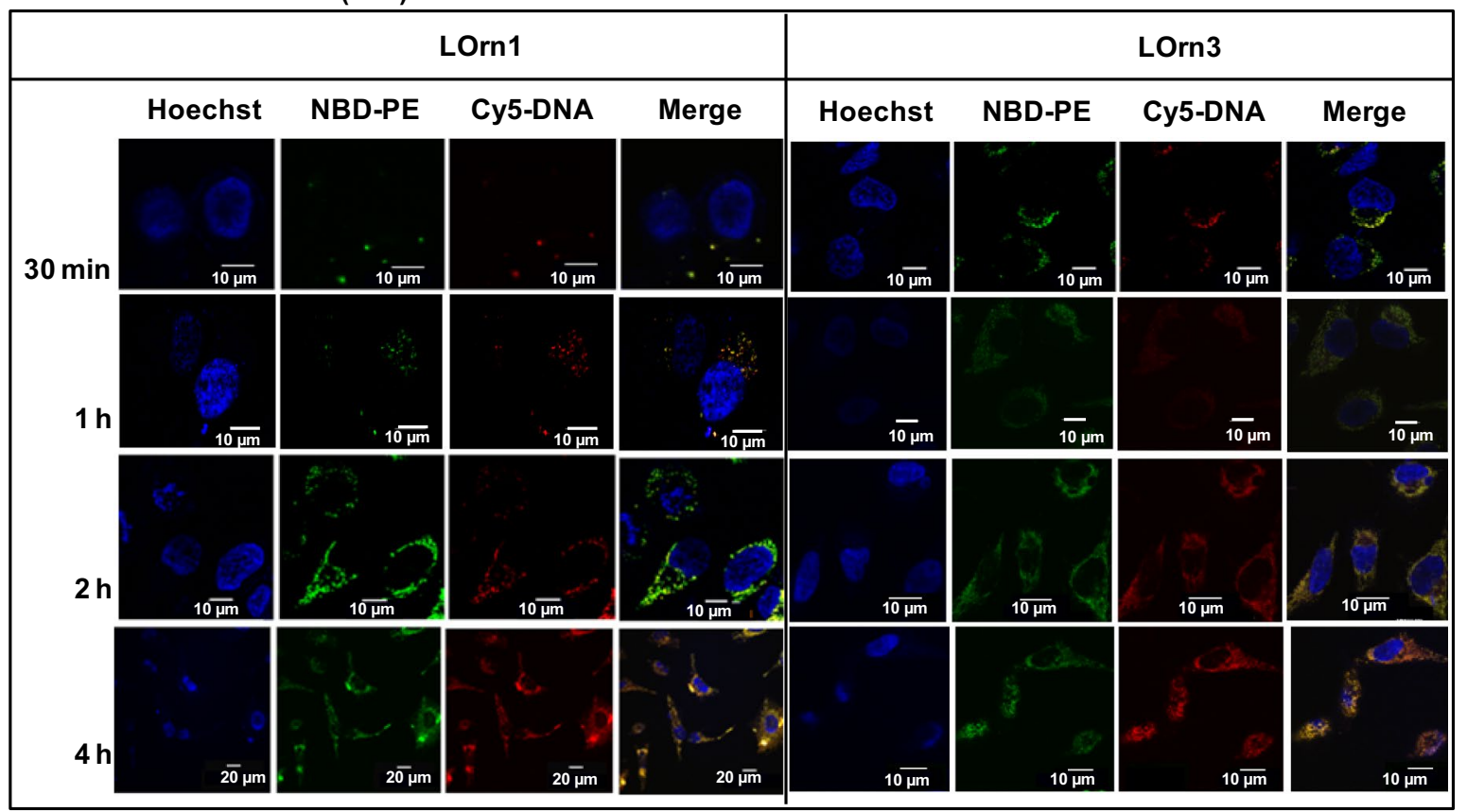

Fig. 7 a Cellular uptake of liposome/FAM-DNA lipoplex in HeLa cells was evaluated by FACS. b Intracellular trafficking of LOrn3/DNA lipoplex in HeLa cells at incubation time of 30 min, 2 h, and 4 h by TEM. c Intracellular tracking of lipoplexes with incubation time of 30 min, 1 h, 2 h, and 4 h in HeLa cells by LSCM. Hoechst was used to label cell nucleus (blue), NBD-PE to label liposomes (green), and Cy5 to label DNA (red)

liposome LOrn3/DNA lipoplexes exhibited a faster and more uniform uptake in HeLa cells. And combined with the interaction data of liposome and DNA (Fig. 5), it's found that the more the liposome-DNA interaction was, the higher the cellular uptake of liposome/DNA lipoplex was.

\section{Dissociation kinetics of liposome and DNA}

After the assembly with DNA, the stimuli-responsive features and DNA dissociation behavior of the lipoplexes were tested in vitro in a simulated intracellular microenvironment $(\mathrm{pH}$ 5-5.5) using a gel retardation assay [35, 36]. As can be seen in Fig. 8a, b, the DNA dissociated from lipoplexes was time-dependent at $\mathrm{pH} 5.5$, and the DNA dissociation rate of the LOrn3/DNA lipoplex was faster than that of the LOrn1/DNA lipoplex. Seventy percent of DNA could dissociate from LOrn3/DNA lipoplex and only $51 \%$ from LOrn $1 /$ DNA lipoplex at $72 \mathrm{~h}$. In contrast, little DNA dissociated from the two lipoplexes at the similar time point $(8 \%$ at $72 \mathrm{~h})$ at $\mathrm{pH} 7.0$. The $\mathrm{pH}$ induced structural destruction of lipoplexes contributed to the DNA release by exposing carbamate bonds in the lipids to the reductive environment for cleavage (Additional file 1: Fig. S4).

A major intracellular barrier for DNA delivery is endosomal entrapment followed by trafficking and lysosomal degradation or exocytosis [37, 38]. The escape of DNA from late endosomes/lysosomes into the cytosol is thought to be a rate-limiting step for many delivery approaches [39]. The lipoplexes are designed to facilitate late endosomal/lysosomal escape of DNA by synergistic effects of the proton sponge effect [40, 41] and the degradation of the peptide lipid LOrn 1 or LOrn 3 by carbamate bond breaks in late endosomes/ 


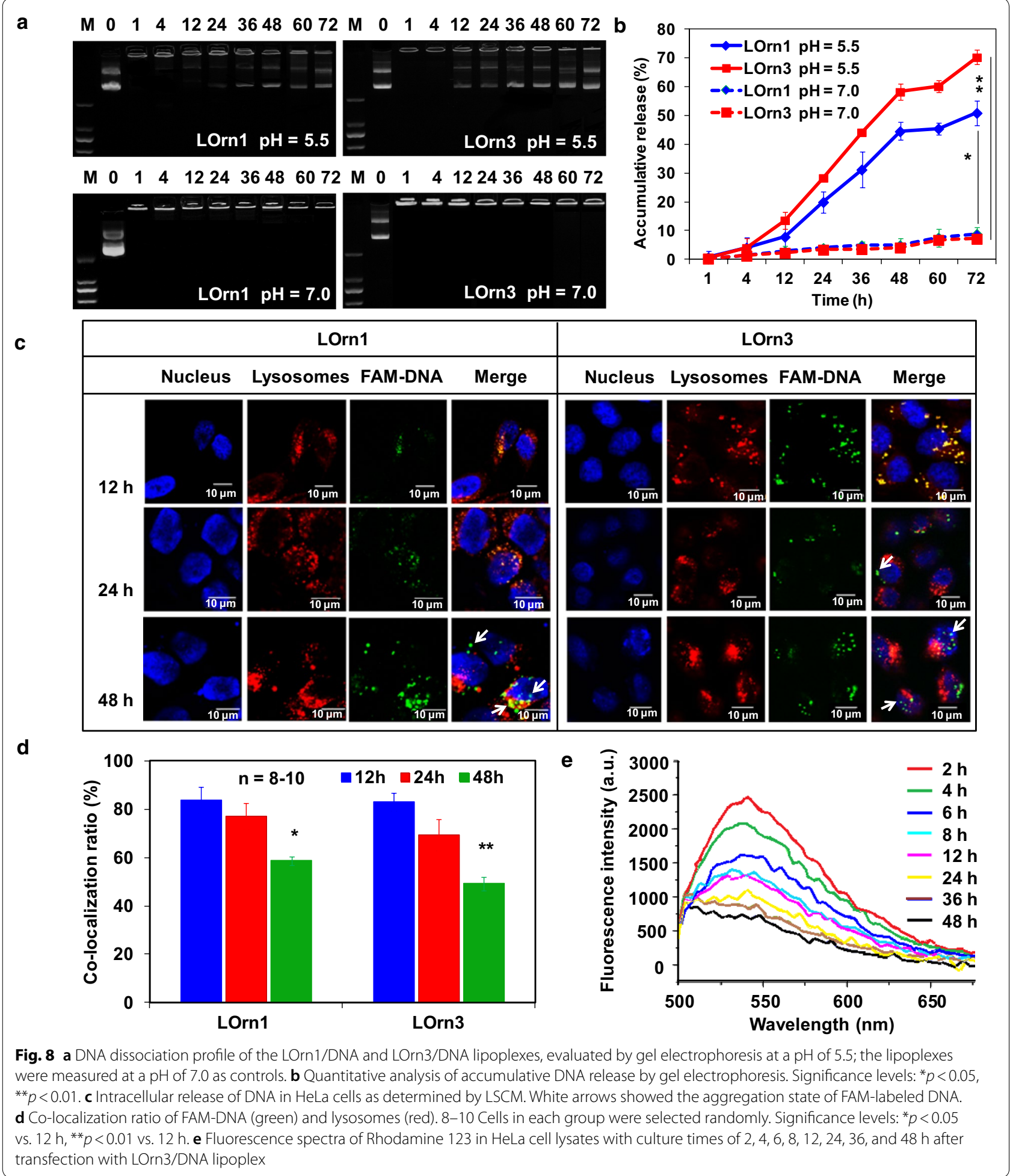

lysosomes. To assess intracellular DNA release, confocal microscopy was used to investigate the distribution of FAM-labeled DNA (green) in lipoplexes and LysoTracker Red labeled late endosomes and lysosomes against HeLa cells. As shown in Fig. 8c, we observed that DNA was gradually released from the both of lipoplexes over time. For LOrn3/DNA lipoplex, most FAM signals of the LOrn3/DNA lipoplex were merged 
in late endosomes/lysosomes at $12 \mathrm{~h}$, as shown by the yellow color. Much DNA could be released from late endosomes/lysosomes at $24 \mathrm{~h}$ after the transfection, and at $48 \mathrm{~h}$, strong green fluorescence in the images for LOrn3/DNA lipoplex showed an obvious release of DNA from late endosomes/lysosomes (indicated with white arrows).

The co-localization ratio (\%) is a commonly used index to measure DNA release $[42,43]$, which was carried out by the following formula:

$$
\text { Co }- \text { localization ratio }(\%)=\frac{\text { Signal }_{\text {yellow }}}{\text { Signal }_{\text {yellow }}+\text { Signal }_{\text {green }}} \times 100
$$

Signal $_{\text {yellow }}$ means the fluorescence signal of FAMDNA (green) co-localized with lysosomes (red), and Signal $_{\text {green }}$ means the fluorescence signal of FAM-DNA in cytoplasm. A low co-localization ratio means quick DNA release from the late endosomes/lysosomes and a better distribution of the DNA in cytoplasm. The results show that the co-localization ratio gradually decreased with time; they were $49 \%$ and $58 \%$ at $48 \mathrm{~h}$ for LOrn 3 and LOrn1, respectively (Fig. 8d). According to the results of the lipoplexes dissociation study in a simulated tumor microenvironment (Fig. 8b), the response to the acidic environment was probably caused by the break of carbamate bonds in the lipids. In addtition, DNA release of LOrn3/DNA lipoplex was more than that of LOrn1/DNA lipoplex at $48 \mathrm{~h}$, this was consistent with the result in Fig. 8b. Although LOrn1 and LOrn3 have a similar chemical constitution, LOrn3, containing more amino groups, promoted not only cellular internalization [44] but also displayed a higher proton buffering capacity. Therefore, LOrn3/DNA lipoplex showed an enhanced capability in late endosomes/lysosomes escape and DNA release compared to LOrn1/DNA lipoplex. That also explains the reason why LOrn3 had more efficient transfection than LOrn1. Accordingly, FRET was utilized to further confirm the intracellular disassembly process of DNA from the LOrn3/DNA lipoplex in HeLa cells, by using Rhodamine-DNA and NBD-PE-LOrn3 liposome to constitute a Rhodamine/NBD FRET system (acceptor/donor) [45]. After the HeLa cells were transfected with lipoplexes for $4 \mathrm{~h}$, they were incubated for $2,4,6,8,12,24,36$, and $48 \mathrm{~h}$. Figure 8e shows the fluorescence emission intensity of Rhodamine-labeled DNA decreased with time. The data indicate that less resonance energy was transferred from donor to acceptor due to lipoplexes dissociation in HeLa cells, and much of the DNA was released from lipoplexes at $48 \mathrm{~h}$ after transfection.

Together, these results strongly demonstrated that both of the lipoplexes could exist in a comparatively stable structure under normal physiological conditions $(\mathrm{pH}$ 7.0), while they rapidly release a significant amount of
DNA due to the destabilization resulting from the acidic microenvironment in endosomes or lysosomes of tumor cells (pH 5.5) [46, 47].

\section{Conclusions}

Our study provides insights into the interaction kinetics, cellular uptake, and dissociation of peptide liposomemediated gene delivery. It highlights similarities and differences between liposomes with either tri-ornithine or mono-ornithine headgroups. We demonstrate that the peptide lipid with three ornithine headgroup (LOrn3) had a strong binding ability to DNA. Moreover, LOrn3/ DNA lipoplex exhibited excellent intracellular stability, effective cellular uptake, lysosomal evasion, and intracellular gene release. Most importantly, we have illustrated the relationship between interaction kinetics and transfection efficiency. In summary, the study proposes a theoretical framework for strategies to improve gene delivery systems.

\section{Supplementary information}

Supplementary information accompanies this paper at https://doi. org/10.1186/s12951-020-00707-1.

Additional file 1: Figure S1. (a) Transfection efficiency of lipoplexes against Hep-2 cells. GFP expression of GFP-N1 was mediated by liposomes LOrn 1 and LOrn3 at the N/P ratios of 1:1, 3:1, 4:1, 6:1 and 8:1. The measurement was carried out in cells by using an inverted fluorescence microscope $(10 \times 10)$. (b) Transfection efficiency in Figure (a) was quantified by flow cytometry analysis. Figure S2. Cell viability assay of HeLa after treating with LOrn1/DNA and LOrn3/DNA lipoplexes for different time by MTT. Figure S3. Small angle x-ray scattering patterns of the lamellar LCa and columnar inverted hexagonal HC\| phases of (a) LOrn1/DNA and (b) LOrn3/DNA lipoplexes at N/P ratios of 4:1 and 3:1, respectively. Figure S4. Effect of acid environment on lipid structure was detected by ESI-MS. (a) ESI-MS analysis of lipid under the pH of 7.0, (b) ESI-MS analysis of lipid under the $\mathrm{pH}$ of 5.5 .

\section{Acknowledgements}

The authors thank for the financial support of National Science Foundation of China (NSFC. 21606041, 21776044, 21908019). Liaoning Provincial Natural Science Foundation (2019-ZD-0185).

\section{Authors' contributions}

YNZ, FLZ, STG and SBZ conceived and designed the study, discussed the results and drafted the manuscript. TYZ performed the SAXA experiment. YYD and YNC performed the cell experiments and analyzed the data. YX contributed to the liposomes preparation for size and zeta measurements. HYC contributed to a part of data analysis work. DFZ contributed to the editing of the manuscript. All authors read and approved the final manuscript.

Availability of data and materials

All data generated or analyzed during this research are included in this article.

Ethics approval and consent to participate

Not applicable within this study.

Consent for publication

Not applicable within this study.

Competing interests

The authors declare that they have no competing interests. 


\begin{abstract}
Author details
${ }^{1}$ Key Laboratory of Biotechnology and Bioresources Utilization of Ministry of Education, College of Life Sciences, Dalian Minzu University, Dalian 116600, China. ${ }^{2}$ School of Materials Science and Engineering, Zhengzhou University, Zhengzhou 450001, China. ${ }^{3}$ Key Laboratory of Functional Polymer Materials of Ministry of Education, State Key Laboratory of Medicinal Chemical Biology and Institute of Polymer Chemistry, College of Chemistry, Nankai University, Tianjin 300071, China. ${ }^{4}$ School of Chemistry and Pharmaceutical Engineering, Jilin Institute of Chemical Technology, Jilin 132022, China.
\end{abstract}

Received: 1 December 2019 Accepted: 9 October 2020

Published online: 17 October 2020

\section{References}

1. Cavazzana-Calvo M, Thrasher A, Mavilio F. The future of gene therapy. Nature. 2004;427:779-81.

2. Kaiser J. Gene therapists celebrate a decade of progress. Science. 2011;334:29-30.

3. Niu J, Chu Y, Huang YF, Chong YS, Jiang ZH, Mao ZW, Peng LH, Gao JQ. Transdermal gene delivery by functional peptide-conjugated cationic gold nanoparticle reverses the progression and metastasis of cutaneous melanoma. ACS Appl Mater Interfaces. 2017:9:9388-401.

4. Wan Y, Dai W, Nevagi RJ, Toth I, Moyle PM. Multifunctional peptide-lipid nanocomplexes for efficient targeted delivery of DNA and siRNA into breast cancer cells. Acta Biomater. 2017;59:257-68.

5. Ashley CE, Carnes EC, Epler KE, Padilla DP, Phillips GK, Castillo RE, Wilkinson DC, Wilkinson BS, Burgard CA, Kalinich RM, Townson JL, Chackerian B, Willman CL, Peabody DS, Wharton W, Brinker CJ. Delivery of small interfering RNA by peptide-targeted mesoporous silica nanoparticle-supported lipid bilayers. ACS Nano. 2012;3:2174-88.

6. Gilleron J, Querbes W, Zeigerer A, Borodovsky A, Marsico G, Schubert U, Manygoats K, Seifert S, Andree C, Stöter M, Epstein-Barash H, Zhang L, Koteliansky V, Fitzgerald K, Fava E, Bickle M, Kalaidzidis Y, Akinc A, Maier M, Zerial M. Image-based analysis of lipid nanoparticle-mediated siRNA delivery, intracellular trafficking and endosomal escape. Nat Biotechnol. 2013;7:638-50.

7. Tagalakis AD, Lee DHD, Bienemann AS, Zhou HY, Munye MM, Saraiva L, McCarthy D, Du ZX, Vink CA, Maeshima R, White EA, Gustafsson K, Hart SL. Multifunctional, self-assembling anionic peptide-lipid nanocomplexes for targeted siRNA delivery. Biomaterials. 2014;35:8406-15.

8. Nie TQ, He ZY, Zhou Y, Zhu JC, Chen KT, Liu LX, Leong KW, Mao HQ, Chen YM. Surface coating approach to overcome mucosal entrapment of DNA nanoparticles for oral gene delivery of glucagon-like peptide 1. ACS Appl Mater Interfaces. 2019;11:29593-603.

9. Writer M, Kyrtatos PG, Bienemann AS, Pugh JA, Lowe AS, Villegas-Llerena C, Kenny GD, White EA, Gill SS, McLeod CW, Lythgoe MF, Hart SL. Lipid peptide nanocomplexes for gene delivery and magnetic resonance imaging in the brain. J Control Release. 2012;2:340-8.

10. Ganguly P, Breen A, Pillai SC. Toxicity of nanomaterials: exposure, pathways, assessment and recent advances. ACS Biomater Sci Eng. 2018:4:2237-75.

11. Gao P, Pan W, Li N, Tang B. Boosting cancer therapy with organelletargeted nanomaterials. ACS Appl Mater Interfaces. 2019;11:26529-58.

12. Hanzlíková M, Ruponen M, Galli E, Raasmaja A, Aseyev V, Tenhu H, Urtti A, Yliperttula M. Mechanisms of polyethylenimine-mediated DNA delivery: free carrier Helps to overcome the barrier of cell-surface glycosaminoglycans. J Gene Med. 2011;13:402-9.

13. Mihaila R, RuHeLa D, Keough E, Cherkaev E, Chang S, Galinski B, Bartz R, Brown D, Howell B, Cunningham JJ. Mathematical modeling: a tool for optimization of lipid nanoparticle-mediated delivery of siRNA. Mol TherNucl Acids. 2017;16:246-55.

14. Rehman Z, Hoekstra D, Zuhorn IS. On the mechanism of polyplex-and lipoplex-mediated delivery of nucleic acids: real-time visualization of transient membrane destabilization without endosomal lysis. ACS Nano. 2013;7:3767-77.

15. Nguyen J, Szoka FC. Nucleic acid delivery: the missing pieces of the puzzle? Accounts Chem Res. 2012;45:1153-62.
16. Silva BFB, Majzoub RN, Chan CL, Li Y, Olsson U, Safinva CR. PEGylated cationic liposome-DNA complexation in brine is pathway-dependent. BBA-Biomembranes. 2014;1838:398-412.

17. Koltover I, Salditt T, Rädler JO, Safinya CR. An inverted hexagonal phase of cationic liposome-DNA complexes related to DNA release and delivery. Science. 1998;281:78-81.

18. Neunert G, Tomaszewska-Gras J, Siejak P, Pietralik Z, Kozak M, Polewski K. Disruptive effect of tocopherol oxalate on DPPC liposome structure: DSC, SAXS, and fluorescence anisotropy studies. Chem Phys Lipids. 2018;216:104-13

19. Zhao YN, Zhang SB, Zhang Y, Cui SH, Chen HY, Zhi DF, Zhen YH, Zhang SF, Huang L. Tri-peptide cationic lipids for gene delivery. J Mater Chem B. 2015:3:119-26.

20. Takahashi T, Harada A, Emi N, Kono K. Preparation of efficient gene carriers using a polyamidoamine dendron-bearing lipid: improvement of serum resistance. Bioconjugate Chem. 2005;16:1160-5.

21. Zhao YN, Liu A, Du YY, Cao YN, Zhang EX, Zhou Q, Hai H, Zhen YH, Zhang SB. Effects of sucrose ester structures on liposome mediated gene delivery. Acta Biomater. 2018;72:278-86.

22. Miteva M, Kirkbride KC, Kilchrist KV, Werfel TA, Li H, Nelson CE, Gupta MK, Giorgio TD, Duvall CL. Tuning PEGylation of mixed micelles to overcome intracellular and systemic siRNA delivery barriers. Biomaterials. 2015;38:97-107.

23. Park K, Yang JA, Lee MY, Lee H, Hahn SK. Reducible hyaluronic acidsiRNA conjugate for target specific gene silencing. Bioconjugate Chem. 2013;24:1201-9.

24. Zhang J, Shrivastava S, Cleveland RO, Rabbitts TH. Lipid-mRNA nanoparticle designed to enhance intracellular delivery mediated by shock waves. ACS Appl Mater Interfaces. 2019;11:10481-91.

25. Gujrati M, Shin MTA, Jin E, Sun Y, Lu ZR. Multifunctional cationic lipidbased nanoparticles facilitate endosomal escape and reduction-triggered cytosolic siRNA release. Mol Pharm. 2014;11:2734-44.

26. Madeira C, Loura LMS, Aires-Barros MR, Fedorov A, Prieto M. Characterization of DNA/Lipid complexes by fluorescence resonance energy transfer. Biophys J. 2003;85:3106-19.

27. Grigsby $\mathrm{CL}$, Ho YP, Leong KW. Understanding nonviral nucleic acid delivery with quantum dot-FRET nanosensors. Nanomedicine. 2012a;4:565-77.

28. Schuette CG, Hatsuzawa K, Margittai M, Stein A, Riedel D, Küster P, König $M$, Seidel $C$, Jahn R. Determinants of liposome fusion mediated by synaptic SNARE proteins. PNAS. 2004;101:2858-63.

29. Marsden HR, Korobko A, Zheng TT, Voskuhl J, Kros A. Controlled liposome fusion mediated by SNARE protein mimics. Biomater Sci. 2013;1:1046-54.

30. Moret I, Peris JE, Guillem VM, Benet M, Revert F, Dasi F, Crespo A, Aliño SF. Stability of PEI-DNA and DOTAP-DNA complexes: effect of alkaline $\mathrm{pH}$, heparin and serum. J Control Release. 2001;76:169-81.

31. Kennedy MT, Pozharski EV, Rakhmanova VA, MacDonald RC. Factors governing the assembly of cationic phospholipid-DNA complexes. Biophys J. 2000;78:1620-33.

32. Jones $\mathrm{CH}$, Chen $\mathrm{C}$, Ravikrishnan A, Rane S, Pfeifer BA. Overcoming nonviral gene delivery barriers: perspective and futur. Mol Pharm. 2013;11:4082-98.

33. Li S, Tseng WC, Stolz DB, Wu SP, Watkins SC, Huang L. Dynamic changes in the characteristics of cationic lipidic vectors after exposure to mouse serum: implications for intravenous lipofection. Gene Ther. 1999;6:585-94.

34. Zhao YN, Zhu J, Zhou HJ, Guo X, Tian T, Cui SH, Zhen YH, Zhang SB, Xu $\mathrm{YH}$. Sucrose ester based cationic liposomes as effective non-viral gene vectors for gene delivery. Colloids Surf, B. 2016;145:454-61.

35. Zhu J, Qiao MX, Wang Q, Ye YQ, Ba S, Ma JJ, Hu HY, Zhao XL, Chen DW. Dual-responsive polyplexes with enhanced disassembly and endosomal escape for efficient delivery of siRNA. Biomaterials. 2018;162:47-59.

36. Hong BJ, Chipre AJ, Nguyen ST. Acid-degradable polymer-caged lipoplex (PCL) platform for siRNA delivery: facile cellular triggered release of siRNA. J Am Chem Soc. 2013;135:17655-8.

37. Yang X, Fan B, Gao W, Li LP, Li TT, Sun JH, Peng XY, Li XY, Wang ZJ, Wang $B Q$, Zhang RP, Xie J. Enhanced endosomal escape by photothermal activation for improved small interfering RNA delivery and antitumor effect. Int J Nanomed. 2018;13:4333-44.

38. Zhou ZW, Zhang QY, Zhang MH, Li HP, Chen G, Qian CG, Oupicky D, Sun MJ. ATP-activated decrosslinking and charge-reversal vectors for siRNA delivery and cancer therapy. Theranostics. 2018;17:4604-19. 
39. Dominska M, Dykxhoorn DM. Breaking down the barriers: siRNA delivery and endosome escape. J Cell Sci. 2010;123:1183-9.

40. Ullah I, Chung K, Beloor J, Kim J, Cho M, Kim N. Trileucine residues in a ligand-CPP-based siRNA delivery platform improve endosomal escape of siRNA. J Drug Target. 2017:4:320-9.

41. Forrest ML, Pack DW. On the kinetics of polyplex endocytic trafficking: implications for gene delivery vector design. Mol Ther. 2002;1:57-66.

42. Han SC, Cheng Q, Wu YD, Zhou JH, Long XW, Wei T, Huang YY, Zheng SQ, Zhang JH, Deng LD, Wang XX, Liang XJ, Cao HQ, Liang ZC, Dong AJ. Effects of hydrphobic core components in amphiphilic PDMAEMA nanoparticles on siRNA delivery. Biomaterials. 2015;48:45-55.

43. Du LL, Wang CR, Meng LW, Cheng Q, Zhou JH, Wang XX, Zhao DY, Zhang $J$ H, Deng LD, Liang ZC, Dong AJ, Cao HQ. The study of relationships between pKa value and siRNA delivery efficiency based on tri-block copolymers. Biomaterials. 2018;176:84-93.

44. Futaki S, Suzuki T, Ohashi W, Yagami T, Tanaka S, Ueda K, Suqiura Y. Arginine-rich peptides: an abundant source of membrane-permbeable peptides having potential as carriers for intracellular protein delivery. J Biol Chem. 2001;8:5836-40.

45. Grigsby $\mathrm{CL}, \mathrm{Ho}$ YP, Leong KW. Understanding nonviral nucleic acid delivery with quantum dot-FRET nanosensors. Nanomedicine. 2012b;7:565-77.

46. Kongkatigumjorn N, Smith SA, Chen M, Fang K, Yang SL, Gillies ER, Johnston APR, Such GK. Controlling endosomal escape using pH-responsive nanoparticles with tunable disassembly. ACS Appl Nano Mater. 2018;7:3164-73.

47. Wang C, Zhao T, Li Y, Huang G, White MA, Gao J. Investigation of endosome and lysosome biology by ultra $\mathrm{pH}$-sensitive nanoprobes. Adv Drug Deliver Rev. 2017;113:87-96.

\section{Publisher's Note}

Springer Nature remains neutral with regard to jurisdictional claims in published maps and institutional affiliations.
Ready to submit your research? Choose BMC and benefit from:

- fast, convenient online submission

- thorough peer review by experienced researchers in your field

- rapid publication on acceptance

- support for research data, including large and complex data types

- gold Open Access which fosters wider collaboration and increased citations

- maximum visibility for your research: over $100 \mathrm{M}$ website views per year

At BMC, research is always in progress.

Learn more biomedcentral.com/submissions 